\title{
Theory of elementary excitations in unstable Bose-Einstein condensates
}

\author{
U. Leonhardt ${ }^{1}$, T. Kiss ${ }^{1,2,3}$, and P. Öhberg ${ }^{1,4}$ \\ ${ }^{1}$ School of Physics and Astronomy, University of St Andrews, \\ North Haugh, St Andrews, KY16 9SS, Scotland \\ ${ }^{2}$ Research Institute for Solid State Physics and Optics, \\ H-1525 Budapest, P. O. Box 49, Hungary \\ ${ }^{3}$ Institute of Physics, University of Pécs, Ifjúság u. 6. H-7624 Pécs, Hungary and \\ ${ }^{4}$ Department of Physics, University of Strathclyde, \\ Glasgow G4 ONG, Scotland
}

\begin{abstract}
Like classical fluids, quantum gases may suffer from hydrodynamic instabilities. Our paper develops a quantum version of the classical stability analysis in fluids, the Bogoliubov theory of elementary excitations in unstable Bose-Einstein condensates. In unstable condensates the excitation modes have complex frequencies. We derive the normalization conditions for unstable modes such that they can serve in a mode decomposition of the non-condensed component. Furthermore, we develop approximative techniques to determine the spectrum and the mode functions. Finally, we apply our theory to a sonic white hole and find that the spectrum of unstable modes is intrinsically discrete.
\end{abstract}

PACS numbers: 03.75.Fi, 04.70.Dy

\section{INTRODUCTION}

Instabilities may haunt classical as well as quantum fluids. For example, classical supersonic flows can trigger shock waves [1] 2] or moving obstacles in Bose-Einstein condensates 3. can shed vortex pairs [4]. In fact, a dynamical instability is at the heart of vortex nucleation in rotating condensates [5]. In classical fluid mechanics [2] the stability of a solution of the equations of motion is treated using stability analysis. Assuming a small perturbation of the solution, the equations are linearized in the perturbation and the eigenvalues of the linearized problem decide the fate of the solution. Complex eigenfrequencies with positive imaginary parts indicate instabilities. In the theory of quantum fluids such as BoseEinstein condensates [3] the equations of motion are linearized around the mean field to find the elementary excitations. The ground state of the condensate is, almost by definition, stable, yet macroscopic flows of condensed atoms may develop instabilities. Here it is important to understand how to test for dynamical instabilities and how unstable fluctuations evolve.

In this paper we elaborate a theory of elementary excitations in unstable Bose-Einstein condensates. Our work is primarily inspired by recent proposals $6,6,8,8,9,10$ for generating analogs of black holes using transsonic condensates, but our theoretical concepts and tools may certainly find applications in other situations as well [11. Surprisingly little systematic work has been published on elementary excitations in unstable condensates, to the best of our knowledge, despite the fundamental nature of the subject. Inspired by the treatment of instabilities in quantum fields [12], Garay et al. studied a quantum theory of instabilities in Bose-Einstein condensates in an appendix [7] and in a brief book contribution [8]. Yurovsky 13] developed an alternative theory of instabilities in quantum fluids. Section II of our paper elaborates on these ideas, starting from the basic concepts of elementary excitations in dilute quantum gases 14. We put forward an economic notation that allows us to derive the theory with as little technical effort as possible. Section III addresses two important approximative methods to describe unstable excitations analytically. We present a brief summary of the frequently applied acoustic approximation and develop a version of the WKB approximation that can be extended to complex frequencies and complex variables. In Section IV we apply all the developed concepts and techniques to the analysis of sonic horizons, demonstrating so their problem-solving potential.

\section{ELEMENTARY EXCITATIONS}

\section{A. Fluctuation field}

Consider a stationary Bose-Einstein condensate of atoms with short-range repulsive interactions. Following Fetter 14 we describe the dynamics of the bosonic atom field $\psi(t, \mathbf{x})$ using the grand-canonical Hamiltonian

$$
\hat{H}-\mu \hat{N}=\int \hat{\psi}^{\dagger}\left(-\frac{\hbar^{2} \nabla^{2}}{2 m}+U-\mu+\frac{1}{2} g \hat{\psi}^{\dagger} \hat{\psi}\right) \hat{\psi} d^{3} x .
$$

Here $\hat{N}$ abbreviates the total number of particles, $\int \hat{\psi}^{\dagger} \hat{\psi} d^{3} x$, a conserved quantity, and $\mu$ denotes a constant, the chemical potential. (Because of particlenumber conservation, both the Hamiltonian $\hat{H}$ and the grand-canonical Hamiltonian (2.1) are equivalent.) We assume that most of the atoms constitute a Bose-Einstein condensate with macroscopic wave function

$$
\psi_{0}=\sqrt{\rho_{0}} e^{i S_{0}}
$$


such that the deviations of $\hat{\psi}$ from the mean field $\psi_{0}$ form a quantum field $\hat{\phi}$ of small fluctuations,

$$
\hat{\psi}=\psi_{0}+e^{i S_{0}} \hat{\phi}
$$

We expand the grand-canonical Hamiltonian (2.1) up to quadratic order in $\hat{\phi}$, and get

$$
\begin{aligned}
\hat{H}-\mu \hat{N}= & \hat{H}_{0}+\hat{H}_{1}+\hat{H}_{2}, \\
\hat{H}_{0}= & \int \psi_{0}^{*}\left(-\frac{\hbar^{2} \nabla^{2}}{2 m}+U-\mu+\frac{1}{2} g \rho_{0}\right) \psi_{0} d^{3} x \\
\hat{H}_{1}= & \int \hat{\phi}^{\dagger}\left(-\frac{\hbar^{2} \nabla^{2}}{2 m}+U-\mu+g \rho_{0}\right) \psi_{0} d^{3} x \\
& + \text { H. c. } \\
\hat{H}_{2}= & \int\left[\hat{\phi}^{\dagger}(\mathcal{T}+U-\mu) \hat{\phi}\right. \\
& \left.\quad+\frac{1}{2} g \rho_{0}\left(4 \hat{\phi}^{\dagger} \hat{\phi}+\hat{\phi}^{\dagger 2}+\hat{\phi}^{2}\right)\right] d^{3} x
\end{aligned}
$$

with the kinetic term

$$
\mathcal{T}=\frac{m}{2}\left(\frac{\hbar \nabla}{i m}+\mathbf{u}\right)^{2}
$$

and the condensate flow

$$
\mathbf{u}=\frac{\hbar}{m} \nabla S_{0}
$$

Here $\hat{H}_{0}$ describes the energy of the condensate. The Hamiltonian $\hat{H}_{1}$ would displace the mean value of the fluctuations, when acting on $\hat{\phi}$, unless we impose the stationary Gross-Pitaevskii equation

$$
\left(-\frac{\hbar^{2} \nabla^{2}}{2 m}+U-\mu-g\left|\psi_{0}\right|^{2}\right) \psi_{0}=0
$$

which minimizes also the Hamiltonian $\hat{H}_{0}$ such that

$$
\hat{H}_{0}=-\frac{1}{2} \int m c^{2} \rho_{0} d^{3} x
$$

Here, and throughout this paper, $c$ denotes the local speed of sound, defined by

$$
m c^{2}=g \rho_{0}
$$

The quadratic Hamiltonian $\hat{H}_{2}$ generates the equations of motion of the fluctuation field $\hat{\phi}$ (Bogoliubov-deGennes equations). We found it advantageous to deviate from the traditional notation of condensate fluctuations [3] and to combine $\hat{\phi}$ and $\hat{\phi}^{\dagger}$ in one spinor field

$$
\hat{\varphi}=\left(\begin{array}{c}
\hat{\phi} \\
\hat{\phi}^{\dagger}
\end{array}\right)
$$

Our spinor representation serves as a convenient shorthand notation, which does not refer to the spin of the atoms of course. In terms of this Bogoliubov spinor $\hat{\varphi}$ the fluctuation field evolves as

$$
\begin{aligned}
i \hbar \partial_{t} \hat{\varphi} & =B \hat{\varphi} \\
B & =\left(T+U-\mu+2 m c^{2}\right) \sigma_{z}+i m c^{2} \sigma_{y} \\
T & =\frac{m}{2}\left(\frac{\hbar \nabla}{i m}+\mathbf{u} \sigma_{z}\right)^{2}
\end{aligned}
$$

where $T$ describes the kinetic energy of the fluctuations. Throughout this paper we use the Pauli matrices in their standard representation

$$
\sigma_{x}=\left(\begin{array}{cc}
0 & 1 \\
1 & 0
\end{array}\right), \sigma_{y}=\left(\begin{array}{cc}
0 & -i \\
i & 0
\end{array}\right), \sigma_{z}=\left(\begin{array}{cc}
1 & 0 \\
0 & -1
\end{array}\right)
$$

The Bogoliubov-deGennes equation (2.11) is nonHermitian, because of the anti-Hermitian spinor-mixing term $i m c^{2} \sigma_{y}$. Therefore, the spectrum of elementary excitations is not necessarily real. Dynamical instabilities may emerge. Finally, as a consequence of Eqs. (2.11), we find

$$
\hat{H}_{2}=\frac{i \hbar}{2} \int\left(\hat{\phi}^{\dagger}\left(\partial_{t} \hat{\phi}\right)-\left(\partial_{t} \hat{\phi}^{\dagger}\right) \hat{\phi}\right) d^{3} x
$$

an expression that we need in the mode expansion of the Hamiltonian $\hat{H}_{2}$.

\section{B. Mode expansion}

As in standard field theories, we expand the Bogoliubov spinor $\hat{\varphi}$ into modes. First we note that $\hat{\varphi}$ is invariant under the conjugation

$$
\overline{\hat{\varphi}} \equiv \sigma_{x} \hat{\varphi}^{\dagger}
$$

Consequently, $\hat{\varphi}$ must have the mode structure

$$
\hat{\varphi}=\sum_{\nu}\left(w_{\nu} \hat{a}_{\nu}+\bar{w}_{\nu} \hat{a}_{\nu}^{\dagger}\right)
$$

The spinor $w_{\nu}$ comprises Bogoliubov's familiar $u_{\nu}$ and $v_{\nu}$ modes [3],

$$
w_{\nu}=\left(\begin{array}{l}
u_{\nu} \\
v_{\nu}
\end{array}\right)
$$

and $\bar{w}_{\nu}$ denotes the conjugated Bogoliubov spinor,

$$
\bar{w}_{\nu}=\sigma_{x} w_{\nu}^{*}=\left(\begin{array}{c}
v_{\nu}^{*} \\
u_{\nu}^{*}
\end{array}\right)
$$

such that [3]

$$
\hat{\phi}=\sum_{\nu}\left(u_{\nu} \hat{a}_{\nu}+v_{\nu}^{*} \hat{a}_{\nu}^{\dagger}\right) .
$$

The mode functions $w_{\nu}$ are subject to the BogoliubovdeGennes equation

$$
i \hbar \partial_{t} w_{\nu}=B w_{\nu}
$$


that implies

$$
i \hbar \partial_{t} \bar{w}_{\nu}=B \bar{w}_{\nu}
$$

In a field theory, modes are orthonormal with respect to an invariant scalar product $\left(w_{1}, w_{2}\right)$ with

$$
\partial_{t}\left(w_{1}, w_{2}\right)=0
$$

in order to guarantee that the $\hat{a}_{\nu}$ and $\hat{a}_{\nu}^{\dagger}$ are annihilation and creation operators. Such a scalar product is

$$
\left(w_{1}, w_{2}\right)=\int w_{1}^{\dagger} \sigma_{z} w_{2} d^{3} x
$$

where $w^{\dagger}$ abbreviates $w^{* T}$. This scalar product is timeinvariant, because

$$
B^{\dagger} \sigma_{z}=\sigma_{z} B
$$

The $\hat{a}_{\nu}$ and $\hat{a}_{\nu}^{\dagger}$ are Bose annihilation and creation operators if we require that

$$
\begin{aligned}
& \left(w_{\nu}, w_{\nu^{\prime}}\right)=\int\left(u_{\nu}^{*} u_{\nu^{\prime}}-v_{\nu^{\prime}}^{*} v_{\nu}\right) d^{3} x=\delta_{\nu \nu^{\prime}}, \\
& \left(\bar{w}_{\nu}, w_{\nu^{\prime}}\right)=\int\left(v_{\nu} u_{\nu^{\prime}}-u_{\nu} v_{\nu^{\prime}}\right) d^{3} x=0 .
\end{aligned}
$$

So far, the mode expansion (2.15) is fairly general. Now consider single-frequency modes,

$$
i \partial_{t} w_{\nu}=\Omega_{\nu} w_{\nu}
$$

We obtain from the invariance (2.21) of the scalar product 2.22

$$
0=i \partial_{t}\left(w_{1}, w_{2}\right)=\left(\Omega_{2}^{*}-\Omega_{1}\right)\left(w_{1}, w_{2}\right) .
$$

Consequently, single-frequency modes are only normalizable when their frequencies $\Omega_{\nu}$ are real $\omega_{\nu}$ [14. In this case we obtain the well-known mode expansion of the Hamiltonian $\hat{H}_{2}$ [14 in terms of independent harmonic oscillators,

$$
\hat{H}_{2}=\sum_{\nu} \hbar \omega_{\nu}\left(\hat{a}_{\nu}^{\dagger} \hat{a}_{\nu}-\int\left|v_{\nu}\right|^{2} d^{3} x\right) .
$$

Single-frequency Bogoliubov spinors with complex $\Omega$, indicating instabilities, have zero norm. However, this fact does of course not prohibit the existence of instabilities. It only means that we must not directly employ such spinors as modes.

\section{Unstable condensates}

Suppose that the frequency of a Bogoliubov spinor $w$ is complex,

$$
\Omega=\omega+i \gamma
$$

Because the Bogoliubov-deGennes equation (2.19) is nonHermitian, each eigenfrequency corresponds to a left and a right eigenfunction, here denoted by $w_{+}$and $w_{l}$, respectively,

$$
B w_{+}=\hbar \Omega w_{+}, \quad w_{l}^{\dagger} B=\hbar \Omega w_{l}^{\dagger} .
$$

As a consequence of the property 2.23 ) we find

$$
B\left(\sigma_{z} w_{l}\right)=\sigma_{z} B^{\dagger} w_{l}=\hbar \Omega^{*}\left(\sigma_{z} w_{l}\right)
$$

Therefore, $\sigma_{z} w_{l}$ is the Bogoliubov spinor with the complex conjugated eigenfrequency of $w_{+}$. Complex frequencies of elementary excitations occur in conjugated pairs, reflecting the Hermiticity of the grand-canonical Hamiltonian (2.1). The spinor conjugate $\bar{w}_{+}$corresponds trivially to the frequency $-\Omega^{*}$, whereas the frequency $-\Omega$ is associated with the spinor

$$
w_{-} \equiv \overline{\sigma_{z} w_{l}}
$$

Consider the scalar product of the modes with $\pm \Omega_{n}$ frequencies that are labeled by the subscripts $\pm n$,

$$
0=i \partial_{t}\left(\bar{w}_{-n}, w_{+n^{\prime}}\right)=\left(-\Omega_{n}+\Omega_{n^{\prime}}\right)\left(\bar{w}_{-n}, w_{+n^{\prime}}\right) .
$$

Consequently, we can require

$$
\left(\bar{w}_{-n}, w_{+n^{\prime}}\right)=\delta_{n n^{\prime}}
$$

by choosing the appropriate overlap between the left and right eigenstates of $B$. In Section IV we use this orthogonality condition to find the unstable elementary excitation of a sonic horizon.

Single-frequency Bogoliubov spinors with complex $\Omega$ must not represent modes per se, yet nothing prevents us from combining two or more of such spinors to form non-stationary modes. A simple choice is

$$
\mathcal{W}_{ \pm n} \equiv \frac{1}{\sqrt{2}}\left(w_{ \pm n} \pm \bar{w}_{\mp n}\right)=\left(\begin{array}{c}
\mathcal{U}_{ \pm n} \\
\mathcal{V}_{ \pm n}
\end{array}\right)
$$

satisfying the relations

$$
\begin{aligned}
& \left(\mathcal{W}_{ \pm n}, \mathcal{W}_{ \pm n^{\prime}}\right)=\delta_{n n^{\prime}},\left(\mathcal{W}_{ \pm n}, \mathcal{W}_{\mp n^{\prime}}\right)=0 \\
& \left(\overline{\mathcal{W}}_{ \pm n}, \mathcal{W}_{ \pm n^{\prime}}\right)=0, \quad\left(\overline{\mathcal{W}}_{ \pm n}, \mathcal{W}_{\mp n^{\prime}}\right)=0
\end{aligned}
$$

Therefore, the $\mathcal{W}_{ \pm n}$ are perfectly suitable as Bogoliubov modes. We expand the fluctuation field $\hat{\phi}$ in terms of the $\mathcal{U}_{ \pm}, \mathcal{V}_{ \pm}$modes

$$
\begin{aligned}
\hat{\phi} & =\sum_{\nu} \hat{\phi}_{n} \\
\hat{\phi}_{n} & =\mathcal{U}_{+n} \hat{a}_{+n}+\mathcal{V}_{+n}^{*} \hat{a}_{+n}^{\dagger}+\mathcal{U}_{-n} \hat{a}_{-n}+\mathcal{V}_{-n}^{*} \hat{a}_{-n}^{\dagger}
\end{aligned}
$$

We obtain from Eqs. (2.25) and 2.28)

$$
\begin{aligned}
& i \partial_{t} \mathcal{U}_{ \pm n}= \pm \omega_{n} \mathcal{U}_{ \pm n}-i \gamma_{n} \mathcal{V}_{\mp n}^{*} \\
& i \partial_{t} \mathcal{V}_{ \pm n}= \pm \omega_{n} \mathcal{V}_{ \pm n}-i \gamma_{n} \mathcal{U}_{\mp n}^{*}
\end{aligned}
$$

and, consequently, 


$$
i \partial_{t} \hat{\phi}_{n}=\left(\omega_{n} \hat{a}_{+n}-i \gamma_{n} \hat{a}_{-n}^{\dagger}\right) \mathcal{U}_{+n}-\left(\omega_{n} \hat{a}_{+n}^{\dagger}+i \gamma_{n} \hat{a}_{-n}\right) \mathcal{V}_{+n}^{*}-\left(\omega_{n} \hat{a}_{-n}+i \gamma_{n} \hat{a}_{+n}^{\dagger}\right) \mathcal{U}_{-n}+\left(\omega_{n} \hat{a}_{-n}^{\dagger}-i \gamma_{n} \hat{a}_{+n}\right) \mathcal{V}_{-n}^{*} .
$$

We insert this result and the expansion (2.36) into formula (2.13), use the orthonormality relations (2.35), and get

$$
\begin{aligned}
\hat{H}_{2}= & \hbar \sum_{n} \omega_{n}\left[\hat{a}_{+n}^{\dagger} \hat{a}_{+n}-\hat{a}_{-n}^{\dagger} \hat{a}_{-n}-\int\left(\left|\mathcal{V}_{+n}\right|^{2}-\left|\mathcal{V}_{-n}\right|^{2}\right) d^{3} x\right] \\
& +\hbar \sum_{n} i \gamma_{n}\left[\hat{a}_{+n} \hat{a}_{-n}-\hat{a}_{+n}^{\dagger} \hat{a}_{-n}^{\dagger}+\int\left(\mathcal{U}_{+n} \mathcal{V}_{-n}-\mathcal{U}_{+n}^{*} \mathcal{V}_{-n}^{*}\right) d^{3} x\right] .
\end{aligned}
$$

Due to the instability of the condensate, pairs of elementary excitations are spontaneously generated at the rates $\gamma_{n}$, and so the non-condensed part grows at the expense of the condensate. Of course, the Hamiltonian $\hat{H}_{2}$ describes the correct dynamics only for short times, as long as the growing excitations are still small compared with the condensate. Furthermore, the backaction of the noncondensed part onto the condensate ought to be taken into account, affecting the growth rates $\gamma_{n}$ and the frequencies $\omega_{n}$. The instability causes the condensate to dissolve. Nevertheless, the atoms may settle afterwards to constitute a new condensate with a stable macroscopic wave function, as happens in vortex nucleation [5].

\section{APPROXIMATIVE METHODS}

\section{A. Acoustic approximation}

Frequently, approximative methods provide the tools to find analytic results that capture the essential physics of elementary excitations. The best known example is the excitation spectrum of a condensate in a harmonic trap [15]. Here the excitations of the condensate have been calculated in hydrodynamic or, as we would prefer to call it, acoustic approximation. (Elementary excitations are sound waves within the validity of the approximation.) Furthermore, sound waves in moving Bose-Einstein condensates propagate in the same way as mass-less waves in a sufficiently large class of curved space-time structures [6, 7, 8, 16]. In Section IV we use this connection to analyze the instabilities of a sonic horizon.

Let us briefly summarize the main aspects of the acoustic approximation. Given a solution (2.16) of the Bogoliubov-deGennes equation (2.19), the function

$$
\psi=\psi_{0}+e^{i S_{0}}\left(u+v^{*}\right)
$$

solves the time-dependent Gross-Pitaevskii equation,

$$
i \hbar \partial_{t} \psi=\left(-\frac{\hbar^{2} \nabla^{2}}{2 m}+U-g\left|\psi_{0}\right|^{2}\right) \psi,
$$

as long as $u$ and $v$ are small. We represent $\psi$ in terms of the particle density $\rho$ and the phase $S$,

$$
\psi=\sqrt{\rho} e^{i S}, \quad \rho=\rho_{0}+\rho_{s}, \quad S=S_{0}+s .
$$

Neglecting the quantum potential $\hbar^{2}\left(\nabla^{2} \sqrt{\rho}\right) /(2 m \sqrt{\rho})$ in the Gross-Pitaevskii equation (3.2), we recover the equation of continuity and the Bernoulli equation,

$$
\begin{aligned}
\partial_{t} \rho+\nabla \cdot\left(\rho \frac{\hbar}{m} \nabla S\right) & =0, \\
\hbar \partial_{t} S+\frac{\hbar^{2}}{2 m}(\nabla S)^{2}+g \rho+U & =0 .
\end{aligned}
$$

Assuming that $\rho_{0}$ and $S_{0}$ satisfy Eq. (3.4) and linearizing in $\rho_{s}$ and $s$ gives

$$
\begin{aligned}
\partial_{t} \rho_{s} & =-\nabla \cdot\left(\rho_{s} \mathbf{u}+\rho_{0} \frac{\hbar}{m} \nabla s\right), \\
\rho_{s} & =-\frac{\hbar \rho_{0}}{m c^{2}}\left(\partial_{t}+\mathbf{u} \cdot \nabla\right) s
\end{aligned}
$$

where $c$ denotes the speed of sound (2.9) and $\mathbf{u}$ describes the flow $(\hbar / m) \nabla S_{0}$. Substituting the expression for $\rho_{s}$ produces the wave equation for sound in irrotational fluids 16, 17]

$$
\begin{aligned}
\partial_{\mu} f^{\mu \nu} \partial_{\nu} s & =0 \\
f^{\mu \nu} & =\frac{\rho_{0}}{c^{2}}\left(\begin{array}{cc}
1 & \mathbf{u} \\
\mathbf{u} & -c^{2} \mathbb{1}+\mathbf{u} \otimes \mathbf{u}
\end{array}\right), \\
\partial_{\nu} & =\left(\partial_{t}, \nabla\right),
\end{aligned}
$$

the central argument in the analogy between sound in moving media and waves in general relativity [8, 16]. Here we have used a relativistic notation with $\mu$ and $\nu$ referring to space-time coordinates (not to the chemical potential of course).

To see how $\rho_{s}$ and $s$ are related to the Bogoliubov spinor we compare $\psi^{2}$ of Eqs. (3.1) and (3.3) to linear order in $\rho_{s}, s, u$ and $v$, and get

$$
u+v^{*}=\sqrt{\rho_{0}}\left(\frac{\rho_{s}}{2 \rho_{0}}+i s\right) .
$$

Consequently, a single-frequency sound wave appears as the excitation mode

$$
\begin{aligned}
& u=\sqrt{\rho_{0}}\left(\frac{\hbar}{2 m c^{2}}(-i \Omega+\mathbf{u} \cdot \nabla)+1\right) i \sigma e^{-i \Omega t}, \\
& v=\sqrt{\rho_{0}}\left(\frac{\hbar}{2 m c^{2}}(-i \Omega+\mathbf{u} \cdot \nabla)-1\right) i \sigma e^{-i \Omega t}(3.1
\end{aligned}
$$


where

$$
s=\sigma e^{-i \Omega t}+\sigma^{*} e^{+i \Omega^{*} t} .
$$

\section{B. WKB approximation}

Another important approximative method to analyze elementary excitations is the WKB approximation [18], the equivalent of semiclassical wave mechanics or geometrical optics (geometrical acoustics). Frequently, the important features of waves are determined by the turning points of rays on the complex plane or by branch points of the momentum. Here we develop a modification of the WKB approximation for elementary excitations [18] that can be analytically continued to complex variables and complex frequencies.

We assume an effectively one-dimensional model with spatial coordinate $z$ and flow $u$. We express the Bogoliubov spinor as

$$
w=\left(w_{0}+\hbar w_{1}+\ldots\right) \exp \left[\frac{i}{\hbar}\left(\int p d z-E t\right)\right]
$$

in terms of the semiclassical momentum $p$ and the energy $E$. We insert the ansatz (3.12) into the BogoliubovdeGennes equation (2.19) and expand the result into powers in $\hbar$. In zeroth order we obtain

$$
\begin{aligned}
B_{0} w_{0}= & E w_{0}, \\
B_{0}= & \left(\frac{1}{2 m}\left(p \mathbb{1}+m u \sigma_{z}\right)^{2}+\left(U-\mu+2 m c^{2}\right)\right) \sigma_{z} \\
& \quad+i m c^{2} \sigma_{y} .
\end{aligned}
$$

The determinant of $B_{0}-E \mathbb{1}$ vanishes when $p$ satisfies the Hamilton-Jacobi equation

$$
\left(\frac{p^{2}}{2 m}+E_{0}\right)^{2}-(E-u p)^{2}=m^{2} c^{4}
$$

with

$$
E_{0}=U+\frac{m}{2} u^{2}-\mu+2 m c^{2} .
$$

The components $u_{0}$ and $v_{0}$ of the envelope $w_{0}$ are linearly dependent, because $w_{0}$ is an eigenstate of $B_{0}$. We get

$$
\begin{aligned}
v_{0} & =\eta u_{0}, \\
\eta & =-\frac{1}{m c^{2}}\left(E_{0}+\frac{p^{2}}{2 m}+u p-E\right) .
\end{aligned}
$$

Before we proceed, let us see how the Hamilton-Jacobi equation is related to the acoustic approximation. The hydrodynamical model (3.4) presumes small local variations in the density and in the flow such that we can describe the condensate locally. Let us assume that the chemical potential $\mu$ corresponds reasonably accurately to the local energy of the condensate, $m c^{2}+U+\frac{m}{2} u^{2}$, such that

$$
E_{0}=m c^{2} .
$$

Then, for $|p| \ll m c$, i.e. for sound with wavelengths much larger than the healing length $\hbar /(\sqrt{2} m c)$ [3], we get the dispersion relation of sound in moving media

$$
(E-u p)^{2}=c^{2} p^{2} .
$$

Additionally, in the relationship (3.16) between the envelope components we ignore the $p^{2} /(2 m)$ term within the acoustic approximation. We get

$$
\eta=-1+\frac{E-u p}{m c^{2}}=-1 \pm \frac{p}{m c},
$$

which represents the linearized Bernoulli equation (3.7) expressed in terms (3.9) of the Bogoliubov-spinor components.

To first order in $\hbar$ we obtain from the BogoliubovdeGennes equation (2.19) and from the ansatz (3.12)

$$
\begin{aligned}
0 & =B_{1} w_{0}+\sigma_{z}\left(B_{0}-E \mathbb{1}\right) w_{1} \\
B_{1} & =\frac{1}{2 i m}\left(\left(2 p \partial_{z}+p^{\prime}\right) \mathbb{1}+m\left(2 u \partial_{z}+u^{\prime}\right) \sigma_{z}\right)
\end{aligned}
$$

For complex frequencies or complex $z$ values the matrix $\sigma_{z}\left(B_{0}-E \mathbb{1}\right)$ is symmetric but not necessarily Hermitian. Since $w_{0}$ is the eigenvector of $\sigma_{z}\left(B_{0}-E \mathbb{1}\right)$ with zero eigenvalue, $B_{1} w_{0}$ must be orthogonal on $w_{0}$ with respect to the scalar product $w_{1}^{T} w_{2}$. Note that this scalar product does not involve complex conjugation. We find

$$
\begin{aligned}
0 & =w_{0}^{T} B_{1} w_{0} \\
& =\frac{1}{2 i m} \partial_{z}\left(\left(u_{0}^{2}+v_{0}^{2}\right) p+m\left(u_{0}^{2}-v_{0}^{2}\right) u\right),
\end{aligned}
$$

which gives the continuity relation

$$
\partial_{z}\left(u_{0}^{2}-v_{0}^{2}\right) v=0
$$

with the velocity

$$
v=\frac{1+\eta^{2}}{1-\eta^{2}} \frac{p}{m}+u=\frac{\partial H}{\partial p}
$$

in terms of the semiclassical Hamiltonian

$$
E=H=u p \pm \sqrt{\left(\frac{p^{2}}{2 m}+E_{0}\right)^{2}-m^{2} c^{4}} .
$$

The continuity relation (3.22) shows how the spinor amplitudes are connected on the complex plane. For real energies and real coordinates $\left(\left|u_{0}\right|^{2}-\left|v_{0}\right|^{2}\right) v$ is exactly conserved, describing a stationary quasiparticle flux [18]. In this case our result agrees with $\left(\left|u_{0}\right|^{2}-\left|v_{0}\right|^{2}\right) v$, apart from an arbitrary phase. On the other hand, for complex energies or complex coordinates $\left(u_{0}^{2}-v_{0}^{2}\right) v$ does not correspond to an exact conservation law in general, but 
still remains a constant within the validity of the WKB approximation. The advantage of our result is that it can be analytically continued such that

$$
u_{0}^{2}-v_{0}^{2}=\frac{A_{0}}{v},
$$

with a constant $A_{0}$ as long as $z$ does not reach the vicinity of a turning point or crosses a Stokes line in the complex plane 19.

\section{Turning points}

At a turning point the velocity $v$ vanishes and, consequently, $u_{0}^{2}-v_{0}^{2}$ diverges such that the WKB approximation is no longer valid in its vicinity. Turning points are the origins of Stokes lines where the WKB solutions are discontinuously connected 19].

Assume that the condensate is nearly uniform around a turning point such that the speed of sound is approximately constant and that the locality condition (3.17) is satisfied. In this case the turning point does not lie at the edge of the condensate, as it is the case for oscillations in harmonic traps 15]. Let us find out whether and where such turning points exist. Our conditions imply that the momentum $p$ depends on $z$ only through the flow $u(z)$. Let us turn matters around and regard $z$ as a function of $u$ and $u$ as a function of $p$. We get from the expression (3.24) of the quasiclassical Hamiltonian

$$
v=\frac{\partial H}{\partial p}=u+\frac{\partial}{\partial p}(E-u p)=-p \frac{\partial u}{\partial p} .
$$

The definition (3.16) of $\eta$ and the Hamilton-Jacobi equation (3.14) with the condition (3.17) implies the relation

$$
\frac{p^{2}}{m^{2} c^{2}}=-\frac{(1+\eta)^{2}}{\eta}
$$

We substitute this result for $p^{2}$ in Eq. (3.16), solve for up, and get

$$
u=\frac{m c^{2}}{p}\left(\varepsilon-\frac{\eta^{2}-1}{2 \eta}\right)
$$

with

$$
\varepsilon=\frac{E}{m c^{2}} .
$$

We differentiate Eq. (3.28) with respect to the momentum $p$, utilize relation (3.27) and its momentum derivative, and arrive at

$$
\frac{\partial u}{\partial p}=\frac{1+(3-2 \varepsilon) \eta+(3+2 \varepsilon) \eta^{2}+\eta^{3}}{2 m(\eta-1)(\eta+1)^{2}} .
$$

The turning points correspond to the zeros $\eta_{0}$ of the numerator. We note that $\varepsilon$ is naturally a small number, because the energy of the elementary excitation ought to be much smaller than the condensate's energy (2.8). We expand $\eta_{0}$ in powers of $\varepsilon^{1 / 3}$,

$$
\eta_{0}=x_{0}+x_{1} \varepsilon^{1 / 3}+x_{2} \varepsilon^{2 / 3}+x_{3} \varepsilon^{3 / 3}+\ldots,
$$

and find the coefficients

$$
x_{0}=-1, x_{1}=\sqrt[3]{-4}, x_{2}=\frac{2}{x_{1}}, x_{3}=-\frac{2}{3} .
$$

Close to a turning point the flow profile depends quadratically on the momentum

$$
u-u_{0}=\left.\frac{\partial^{2} u}{\partial p^{2}}\right|_{0} \frac{\left(p-p_{0}\right)^{2}}{2} .
$$

This relation shows that a turning point is a branch point for the semiclassical momentum $p$ and it also specifies the onset of Stokes lines [19], defined as the lines where the differences between the phases $\int p d z$ of the two $p$ branches is purely imaginary. Here one of the waves is exponentially small compared with the other. Crossing a Stokes line connects waves in a discontinuous yet precisely defined way 19]. We obtain the coefficients from Eqs. (3.27) and (3.30), using the series (3.31),

$$
\begin{aligned}
\pm \frac{p_{0}}{m c} & =x_{1} \varepsilon^{1 / 3}-\frac{\varepsilon}{6}+\ldots \\
\pm\left.\frac{\partial^{2} u}{\partial p^{2}}\right|_{0} & =-\frac{3}{4}-\frac{5}{8 x_{1}} \varepsilon^{2 / 3}-\frac{\varepsilon}{4}+\ldots
\end{aligned}
$$

Finally, we determine the velocity $u_{0}$ at a turning point from Eq. (3.28). We find

$$
\pm \frac{u_{0}}{c}=-1+\frac{3}{2} \sqrt[3]{-1}\left(\frac{\varepsilon}{2}\right)^{2 / 3}+\mathrm{O}\left(\varepsilon^{4 / 3}\right) .
$$

Here $\sqrt[3]{-1}$ refers to the three cubic roots of -1 , generating three turning points in the complex plane when $u$ approaches $c$. In a mostly uniform condensate the turning points of elementary excitation are close to transsonic regions where the condensate flow transcends the speed of sound. Such a region forms a sonic horizon.

\section{SONIC HORIZONS}

\section{A. Model}

Consider a stationary transsonic medium i.e. a medium with a spatially non-uniform flow that varies from subsonic to supersonic speed. One would expect that beyond the interface where the flow exceeds $c$ sound waves are swept away such that no sound from the supersonic zone can return to the subsonic region. This transsonic interface serves as the sonic equivalent of a black-hole horizon. [6, 7, 8, 16]. On the other hand, the interface where the flow settles from supersonic to subsonic speed forms the horizon of a sonic white hole 
[6, 7, 8], an object that no sound wave can enter from outside. Close to the horizons, sound waves propagating against the current freeze, and their wavelengths are dramatically reduced.

Transsonic Bose-Einstein condensates offer great prospects [6, 7, 8, 9, 10] for demonstrating the quantum effects of event horizons [20, 21]. Such effects and the stability of the condensate depend on the behaviour of the condensate close to the horizons. Let us thus focus on the physics in the vicinity of a black- or white-hole horizon. In this case we can use the simple one-dimensional model

$$
u=-c+\alpha z .
$$

Here $z$ denotes the spatial coordinate orthogonal to the horizon at $z=0, \alpha$ characterizes the surface gravity or, in our acoustic analog, the gradient of the transsonic flow, and $\rho_{0}$ and $c$ are assumed to be constant. Strictly speaking, we should complement the flow component (4.1) in the $z$ direction by appropriate components in the $x$ and $y$ directions, in order to obey the continuity of the flow. But as long as we focus on effects on length scales smaller than $|c / \alpha|$ we can ignore the other dimensions of the fluid. Depending on the sign of $\alpha$, two cases emerge. When the velocity gradient is positive we are considering the horizon of a sonic black hole. When $\alpha$ is negative the horizon refers to a white hole [6, 7, 8]. In a typical alkali Bose-Einstein condensate without exploitation of Feshbach resonances the speed of sound $c$ is in the order of $1 \mathrm{~mm} / \mathrm{s}$. The transsonic velocity gradient should be small compared with the healing length [3]

$$
|\alpha| \xi \ll c, \quad \xi=\frac{\hbar}{m c \sqrt{2}},
$$

which guarantees that the Hawking energy $k_{B} T_{H}$ is much smaller than the energy of the condensate,

$$
k_{B} T_{H}=\frac{\hbar|\alpha|}{2 \pi} \ll m c^{2} .
$$

Being in the vicinity of the horizon and having the linear velocity profile (4.1) presumes that

$$
|z| \ll\left|\frac{c}{\alpha}\right| .
$$

The energies of elementary excitations should be sufficiently smaller than $m c^{2}$, which implies that their dimensionless energy parameter $\varepsilon$ defined in Eq. (3.29) is small. The excitations are sound waves for low wavenumbers and for $z$ far away from the turning points,

$$
|z| \gg\left|z_{0}\right|, \quad z_{0}=\frac{3 c}{2 \alpha} \sqrt[3]{-1}\left(\frac{\varepsilon}{2}\right)^{2 / 3} .
$$

Given the linear velocity profile (4.1), we solve the wave equation (3.8) exactly, and get

$$
s=s_{0}\left(i z^{ \pm i \Omega / \alpha} e^{-i \Omega t}-i z^{\mp i \Omega^{*} / \alpha} e^{i \Omega^{*} t}\right) .
$$

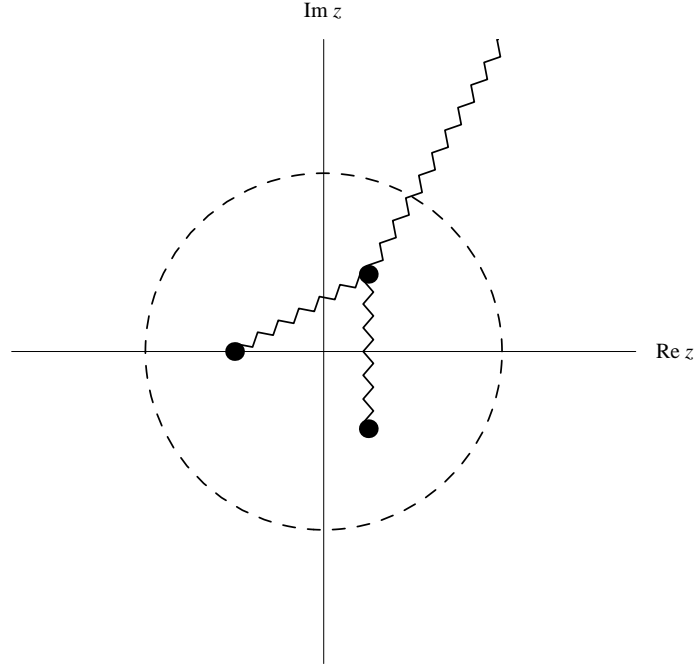

FIG. 1: Analytic structure of a sonic horizon in a BoseEinstein condensate. The figure shows the three turning points in the complex $z$ plane around the horizon at the origin. The points are connected by two branch cuts where the wavenumber $k$ of acoustic elementary excitations is elevated to other solutions of the dispersion relation. The circle around the turning points roughly indicates the place where $k$ approaches the asymptotics $\Omega /(\alpha z)$ that is characteristic of waves at a horizon 21. The third branch cut, connecting one of the turning points to infinity, is the cut between the two trans-acoustic branches with the asymptotics (4.11).

We use the relationship (3.10) to find the Bogoliubov spinor within the acoustic approximation,

$$
w=A\left(\begin{array}{c}
\frac{\Omega}{2 \alpha z}+\frac{m c}{\hbar} \\
\frac{m c}{2 \alpha z}-\frac{m c}{\hbar}
\end{array}\right) z^{ \pm i \Omega / \alpha} e^{-i \Omega t},
$$

where

$$
A=-s_{0} \frac{\hbar \sqrt{\rho_{0}}}{m c} .
$$

The approximation (4.7) is restricted to complex $z$ variables outside the trans-acoustic region indicated in Fig. 1. Close to the trans-acoustic zone the wavelength of elementary excitations is dramatically reduced. In this regime we can use the WKB approximation. In the region where both the acoustic and the WKB approximation are applicable we represent the Bogoliubov spinor as

$$
w=\left(\begin{array}{c}
u_{0} \\
v_{0}
\end{array}\right) \exp \left(i \int k d z-i \Omega t\right)
$$

with the wavenumber

$$
k \sim \frac{\Omega}{\alpha z} .
$$

If the acoustic approximation were universally valid the wavenumber $k$ would approach a singularity at the horizon where, consequently, the wavelength of sound would 

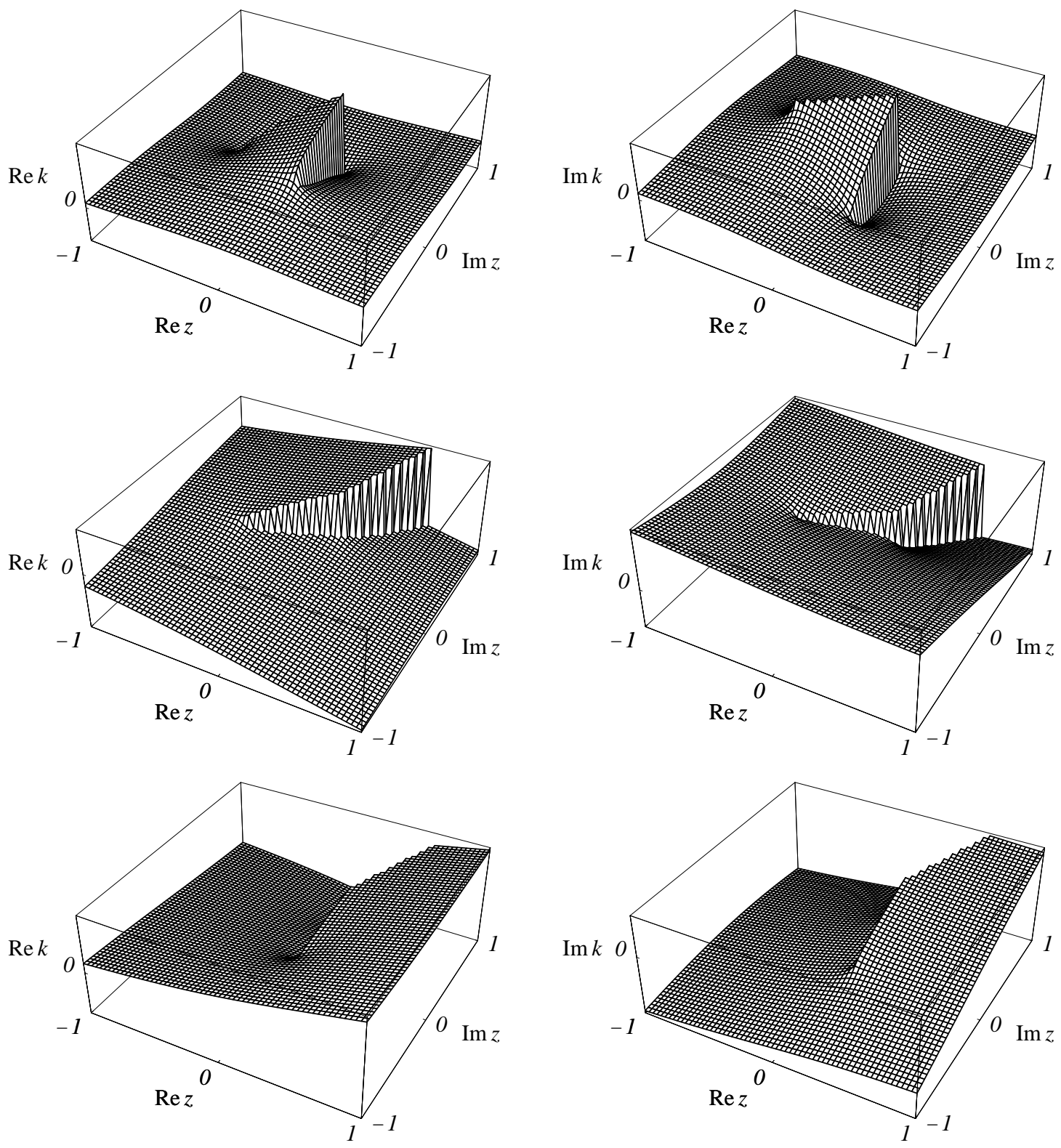

FIG. 2: Wavenumbers $k$ of elementary excitations around a sonic white-hole horizon, analytically continued on the complex plane. The figure shows three roots of the dispersion relation $\left[\hbar^{2} k^{2} /(2 m)+m c^{2}\right]^{2}-\hbar^{2}[\Omega-(-c+\alpha z) k]^{2}=m^{2} c^{4}$ for $\Omega=$ $0.1 i\left(m c^{2} / \hbar\right)$ and $\alpha=-0.5\left(m c^{2} / \hbar\right)$, illustrating the branch cuts of $k$. The top row displays the wavenumber of a sound wave that propagates against the current. The picture indicates the characteristic $\Omega /(\alpha z)$ asymptotics away from the branch points. The two lower rows display two trans-acoustic branches of $k$. The fourth root of the dispersion relation is not shown, because it corresponds to the trivial case of sound waves that propagate with the flow.

shrink to zero. Apparently, Nature tends to prevent such extreme behaviour [22]. In our case, the singularity is split into three branch points, the turning points. The branch cuts between the points connect the acoustic branch to two other trans-acoustic branches with wavenumbers so high that the branches do not repre- sent sound waves subject to the wave equation (3.8). We solve the Hamilton-Jacobi equation (3.14) in the $|\Omega| \ll|u k|$ limit and get the asymptotics for the transacoustic branches 23]

$$
k \sim \pm 2 \frac{m c}{\hbar} \sqrt{u^{2} / c^{2}-1}+\frac{\Omega u}{c^{2}-u^{2}} .
$$


Figure 2 illustrates the momenta $k$ of the three branches in the complex $z$ plane. Finally, we note that the fourth solution of the Hamilton-Jacobi equation (3.14) corresponds to sound waves that are swept away by the current, a less interesting case.

\section{B. Stokes phenomenon}

It is tempting to assume that we could employ the acoustic asymptotics $\Omega /(\alpha z)$ of the wavenumber $k$ on the entire complex plane, as long as $z$ is sufficiently far away from the turning points at the horizon. In this case, however, the phase $\int k d z$ becomes logarithmic and hence multivalued, whereas the true Bogoliubov mode function is singlevalued. The Stokes phenomenon 19 resolves this conflict by connecting the acoustic modes to trans-acoustic ones on the upper or the lower half plane, giving rise to connection formulas [23] that describe mode conversion 24]. Each turning point $z_{0}$ connects two branches of WKB wavenumbers, as we see from Eq. (3.33), and each $z_{0}$ is origin of three Stokes lines. At a Stokes line the phase difference between the two connected branches is purely imaginary. Consequently, one of the WKB waves exponentially exceeds the other such that the smaller cannot be resolved within the WKB approximation, if the larger wave is present. In general, the Bogoliubov spinor is a superposition of the four fundamental solutions that correspond to the four branches of the WKB wavenumbers.

$$
w=c_{A} w_{A}+c_{B} w_{B}+c_{C} w_{C}+c_{D} w_{D} .
$$

When crossing a Stokes line, the exponentially suppressed solution may gain an additional component that is proportional to the coefficient of the exponentially enhanced solution. If we wish to construct a Bogoliubov spinor where only the exponentially smaller component exits in the vicinity of a Stokes line we must put the coefficient of the larger one to zero. Figure 3 shows the Stokes lines of Boboliubov modes at a sonic white hole with purely imaginary frequency, a case of importance in Sec. IV C. The pairs of letters indicate which branches are connected by the lines, and the first letter identifies the exponentially dominant branch. The picture shows that with the choice of branch cuts made we can construct a Bogoliubov mode that is acoustic on the lower half plane, by demanding that $c_{C}$ vanishes at the C,A Stokes line of the lowest turning point and that $c_{B}$ is zero on the lower half plane. Since $c_{D}$ is not connected to the other three branches we can put the coefficient to zero throughout the complex plane. Such a strategy is not possible for a sonic black hole, see Fig. 4, because here the order of exponentially dominant or suppressed waves is exchanged. Moreover, we cannot find a solution that is purely acoustic on the upper half plane, because of the branch cut between the central and the highest turning point in Fig. 4. We can of course alter the arrangements of branch cuts, such that the cut between the two trans-acoustic

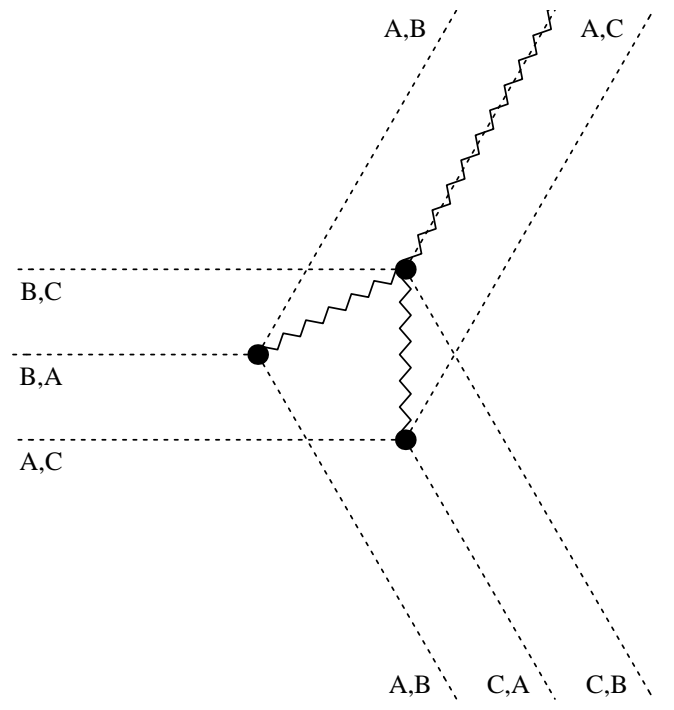

FIG. 3: Stokes lines of elementary excitations at a sonic white hole with purely imaginary frequencies (dotted lines). The pairs of letters indicate which branches of the superposition (4.11) are connected by the lines. The first letter of each pair identifies the exponentially dominant branch.

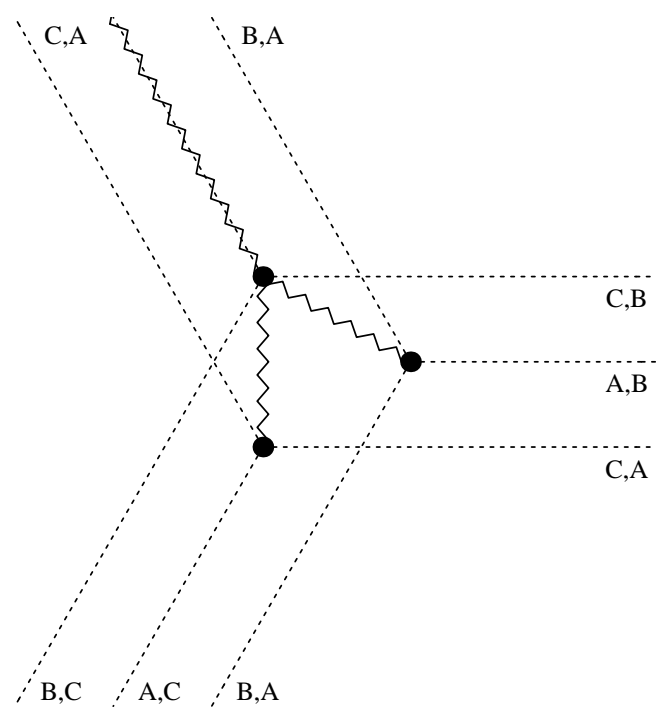

FIG. 4: Stokes lines of elementary excitations at a sonic black hole with purely imaginary frequencies analogous to Fig. 3.

branches $\mathrm{B}$ and $\mathrm{C}$ points in other directions. Choosing different branch cuts does determine in which half plane the excitation wave may be acoustic, but it does not influence whether such an acoustic behavior is possible at all on either the upper or the lower half plane. Therefore, at a sonic white hole unstable elementary excitations with the acoustic asymptotics (4.7) exists on one of the complex half planes, whereas the unstable modes of black holes, if any, are always trans-acoustic. 


\section{Instabilities}

Consider the unstable elementary excitations of a sonic white hole, assuming the acoustic asymptotics (4.7) with complex frequencies $\omega+i \gamma$ to be valid on one of the complex half planes. Excitations (4.7) with negative $\gamma$ are localized near the horizon and are attenuated in time, whereas acoustic excitations with positive $\gamma$ would grow in space and time. Therefore, the truly unstable modes are trans-acoustic. Nevertheless, we can use the properties of the attenuated modes to determine the spectrum of unstable modes, because of the spectral symmetry of the Bogoliubov-deGennes equations proven in Sec. II C.

Bogoliubov excitations with complex frequencies have zero norm and should satisfy the orthogonality relation (2.33). First we calculate the scalar product $\left(w_{1}, w_{2}\right)$ at time $\tau=0$, to find a condition for zero norm. We assume that the scalar product $(2.22)$ is dominated by the acoustic region where $\left|z_{0}\right| \ll|z| \ll|c / \alpha|$,

$$
\begin{aligned}
\left(w_{1}, w_{2}\right)= & \int w_{1}^{\dagger} \sigma_{z} w_{2} d z \\
\sim & |A|^{2} \frac{m c}{\hbar}\left(\int_{-\infty}^{0}+\int_{0}^{+\infty}\right) \frac{2 \omega}{\alpha z} z^{\left(\omega_{1}^{*}-\omega_{2}\right) / \alpha} d z \\
= & |A|^{2} \frac{m c}{\hbar}\left(-e^{ \pm 2 \pi \omega / \alpha}+1\right) \\
& \times \int_{0}^{\infty} \frac{2 \omega}{\alpha z} \exp \left(i \frac{\omega_{1}^{*}-\omega_{2}}{\alpha} \ln z\right) d z
\end{aligned}
$$

Here we have connected the two acoustic regions on the upper or lower half plane, respectively, indicated by the \pm sign, circumventing the trans-acoustic region close to the horizon at $z=0$. The scalar product vanishes if

$$
\omega=0 \text {. }
$$

Therefore, within the validity range of our approximations, the unstable elementary excitations have purely imaginary frequencies $\gamma$. Let us examine the orthogonality condition (2.33). We represent $\gamma$ as

$$
\gamma=2 n \alpha
$$

and we deform the integration contour such that it circumvents the trans-acoustic zone on a large semi-circle with radium $r$ from below.

$$
\begin{aligned}
\left(\bar{w}_{-n}, w_{+n^{\prime}}\right)= & \int\left(v_{-n} u_{+n^{\prime}}-u_{-n} v_{+n^{\prime}}\right) d z \\
\sim & A_{-n} A_{+n^{\prime}} \frac{m c}{\hbar} \int \frac{2 n+2 n^{\prime}}{i z} z^{2\left(n-n^{\prime}\right)} d z \\
= & A_{-n} A_{+n^{\prime}} \frac{m c}{\hbar}\left(2 n+2 n^{\prime}\right) r^{2\left(n-n^{\prime}\right)} \\
& \times \int_{\pi}^{2 \pi} e^{2 i\left(n-n^{\prime}\right) \theta} d \theta
\end{aligned}
$$

The scalar product $\left(\bar{w}_{-n}, w_{+n^{\prime}}\right)$ vanishes for $n \neq n^{\prime}$ if the $n$ are integers. The spectrum of unstable elementary excitations consists of discrete and equally spaced points on the imaginary frequency axis.

An interesting proposal for a sonic hole [6, 6, 8] involves a toroidal condensate that flows through a constriction where it exceeds the speed of sound and that then, after the constriction, settles to subsonic speed. Garay et al. [6, 7, 8] found that the condensate is unstable only in narrow "instability fingers" in the parameter space used. Our analysis indicates that the instabilities are generated when the excitations of the toroidal condensate match the discrete imaginary resonances of the white hole. This would explain the narrowness of the instability fingers [6, 7, 8]. Such instabilities are enhanced by the "lasing" effect of the black-hole white-hole pair 24]. The white hole generates a hydrodynamic instability that is resonantly enhanced by the pair of horizons where elementary excitations can bounce back and fourth. Sonic black holes can be stabilized by employing the equivalent of a Laval nozzle [9] that converts a subsonic flow to a supersonic one without causing turbulence (as in a rocket engine). Our theory indicates that white holes are intrinsically unstable [25], generating breakdown shocks [1].

\section{SUMMARY}

Unstable Bose-Einstein condensates develop elementary excitations with complex frequencies. Such excitations have zero norm and are subject to orthogonality relations between pairs of excitations with opposite frequencies. We elaborated the general theory of unstable elementary excitations and of two important approximate methods to analyze their behaviour, the acoustic and the WKB approximations. Applying these techniques, we showed that sonic white holes in Bose-Einstein condensates give rise to a discrete spectrum of instabilities, which may explain the remarkable stability of sonic holes in toroidal traps [6, 元, 8].

\section{ACKNOWLEDGEMENTS}

We thank J. R. Anglin, M. V. Berry, I. BialynickiBirula, I. A. Brown, L. J. Garay, T. A. Jacobson, R. Parentani, S. Stenholm, M. Visser, and G. E. Volovik for discussions. Our work was supported by the ESF Programme Cosmology in the Laboratory, the Leverhulme Trust, the National Science Foundation of Hungary (contract No. F032346), the Marie Curie Programme of the European Commission, the Royal Society of Edinburgh, and by the Engineering and Physical Sciences Research Council. 
[1] R. Courant and K. O. Friedrichs, Supersonic Flow and Shock Waves (Wiley, New York, 1967).

[2] L. D. Landau and. E. M. Lifshitz, Fluid Mechanics (Pergamon, Oxford, 1982).

[3] F. Dalfovo, S. Giorgini, L. P. Pitaevskii, and S. Stringari, Rev. Mod. Phys. 71, 463 (1999).

[4] C. Raman, M. Köhl, R. Onofrio, D. S. Durfee, C. E. Kuklewicz, Z. Hadzibabic, and W. Ketterle, Phys. Rev. Lett. 83, 2502 (1999); S. Inouye, S. Gupta, T. Rosenband, A. P. Chikkatur, A. Görlitz, T. L. Gustavson, A. E. Leanhardt, D. E. Pritchard, and W. Ketterle, ibid. 87, 080402 (2001).

[5] S. Sinha and Y. Castin, Phys. Rev. Lett. 87, 190402 (2001).

[6] L. J. Garay, J. R. Anglin, J. I. Cirac, and P. Zoller, Phys. Rev. Lett. 85, 4643 (2000).

[7] L. J. Garay, J. R. Anglin, J. I. Cirac, and P. Zoller, Phys. Rev. A 63, 023611 (2001).

[8] L. J. Garay, Acoustic black holes in dilute Bose-Einstein condensates in Artificial Black Holes edited by M. Novello, M. Visser, and G. E. Volovik (World Scientific, Singapore, 2002).

[9] C. Barcelo, S. Liberati, and M. Visser, arXiv: grqc/0110036; M. Sakagami and A. Ohashi, Prog. Theor. Phys. 107, 1267 (2002).

[10] U. Leonhardt, T. Kiss, and P. Öhberg, arXiv:condmat/0211464.

[11] One of the most spectacular unstable Bose-Einstein condensates is the Bose nova, see E. A. Donley, N. R. Claussen, S. L. Cornish, J. L. Roberts, E. A. Cornell, C. E. Wieman, Nature (London) 412295 (2001). Here a condensate collapses due to a change from repulsive to attractive atom-atom interaction, induced by a Feshbach resonance. Our theory can be easily modified to describe the initial stage of a collapsing condensate with attractive interactions.

[12] See G. Kang, Phys. Rev. D 55, 7563 (1997) and references therein, especially S. A. Fulling, Aspects of Quan- tum Field Theory in Curved Spacetime (Cambridge University Press, Cambridge, 1989); B. Schroer and J. A. Swieca, Phys. Rev. D 2, 2938 (1970).

[13] V. A. Yurovsky, Phys. Rev. A 65, 033605 (2002).

[14] A. L. Fetter, Ann. Phys. (N.Y.) 70, 67 (1972); Phys. Rev. A 53, 4245 (1996).

[15] S. Stringari, Phys. Rev. Lett. 77, 2360-2363 (1996); M. Fliesser, A. Csordás, P. Szépfalusy, and R. Graham Phys. Rev. A 56, R2533 (1997); P. Öhberg, E. L. Surkov, I. Tittonen, S. Stenholm, M. Wilkens, and G. V. Shlyapnikov, ibid., R3346 (1997).

[16] W. G. Unruh, Phys. Rev. Lett. 46, 1351 (1981); M. Visser, Class. Quantum Grav. 15, 1767 (1998).

[17] For a wave equation of sound in fluids with vorticity see S. E. P. Bergliaffa, K. Hibberd, M. Stone, and M. Visser, cond-mat/0106255.

[18] A. Csordás, R. Graham, and P. Szépfalusy, Phys. Rev. A 57, 4669 (1998).

[19] M. J. Ablowitz and A. S. Fokas, Complex Variables (Cambridge University Press, Cambridge 1997); R. B. Dingle, Asymptotic Expansions: Their Derivation and Interpretation (Academic, London, 1973); W. H. Furry, Phys. Rev. 71, 360 (1947).

[20] S. M. Hawking, Nature (London) 248, 30 (1974); Commun. Math. Phys. 43, 199 (1975).

[21] R. Brout, S. Massar, R. Parentani, and Ph. Spindel, Phys. Rep. 260, 329 (1995).

[22] The issue is related to the trans-Planckian problem of black holes, see G. t'Hooft, Nucl. Phys. B 256, 727 (1985); T. Jacobson, Phys. Rev. D 44, 1731 (1991); W. G. Unruh, Phys. Rev. D 51, 2827 (1995); R. Brout, S. Massar, R. Parentani, and Ph. Spindel, ibid.52, 4559 (1995).

[23] S. Corley, Phys. Rev. D 57, 6280 (1998).

[24] S. Corley and T. Jacobson, Phys. Rev. D 59, 124011 (1999).

[25] U. Leonhardt, T. Kiss, and P. Öhberg, gr-qc/0211069. 